\title{
Quality improvement at times of crisis
}

\author{
Making rapid change during covid-19 has lessons for how to improve healthcare, argue Amar Shah \\ and colleagues
}

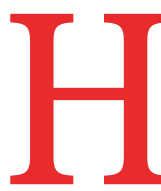

ealth systems across the globe have faced unprecedented strain and uncertainty during the coronavirus pandemic. Healthcare providers have had to respond rapidly, making major changes to all aspects of healthcare from moving to remote delivery of primary care services to creating covid "hot" and "cold" zones and developing innovations which are likely to have both short and long term consequences for the health of the population.

Quality improvement has been increasingly used globally over the past decade to change healthcare. Evidence of success is mixed. ${ }^{1}$ Have such approaches added any value to healthcare service delivery during these unparalleled rapid changes? Are scientifically based approaches to complex system change, such as quality improvement, helping healthcare providers during a crisis?

Several authors have suggested ways in which quality improvement might be applied during the pandemic-for example, by generating rapid learning about which changes are effective when many changes

\section{KEY MESSAGES}

- Quality improvement tools have been applied during the covid-19 pandemic in a range of healthcare settings to standardise and improve processes, to measure change, and to test and implement intervention

- An opportunity exists to build on the widespread change that has been led by healthcare staff at the point of care and embed the rigour of quality improvement without the jargon and bureaucracy that might have stifled past efforts

- Approaches to improvement offer valuable ways for dealing with complex problems, even in pressurised situations

- Sustained success is likely to require commitment to the chosen improvement method and for tools to be seen as part of a discipline of continuous improvement are happening simultaneously ${ }^{2}$ and through generating, testing, refining, and evaluating particular ideas speedily. Quality improvement could enhance workflow, provide a systematic approach to change, strengthen the system, and increase learning. $^{34}$

This paper offers evidence based on personal experience of how quality improvement has been applied during the pandemic and how it has contributed to the response, and reflects on what we might learn.

\section{Has quality improvement been used during the covid-19 pandemic?}

A survey of 225 Q community members (people involved in improving health and care across the United Kingdom ${ }^{5}$ ) suggests that approaches to improvement have been used widely during the pandemic. Fifty one per cent of respondents said that quality improvement had been important during covid-19. A considerable number of respondents felt that the role of improvement tools, methods, approaches, or attitudes increased in their work (44\% of all respondents), their team (45\%), and their organisation (49\%).

Below, we describe five ways in which attempts to improve quality have been made during the covid-19 pandemic.

\section{Creating a common theory about how to tackle} complex problems

Healthcare organisations and leaders have been faced with managing a disease with unknown clinical characteristics. Creating a coherent response within a health service was critical for a major and speedy reorganisation of service delivery and to protect staff from infection. Driver diagrams are a tool commonly used to describe the theory of change for solving a complex problem. These diagrams can help a diverse group of people, who may be working outside their established roles, to collaborate in developing change that will achieve a shared goal. ${ }^{6}$

East London NHS Foundation Trust, a UK provider of mental health, community health, and primary care, developed a driver diagram to help organise its response to phase 2 of the pandemic (fig 1). Developing the driver diagram involved a range of participants, including service users, clinicians, and senior leaders. Using an approach to tackling the problem which was already embedded in organisational practice provided people with a familiar method of dealing with complex problems, thereby providing continuity of approach. The driver diagram also helped leadership to communicate the way in which the organisation was planning its response.

The Albert Einstein Hospital in Sao Paulo, Brazil (fig 2), similarly developed a driver diagram. The hospital asked staff about barriers and fears during the pandemic to ascertain how the organisation could reduce harm for staff. As of April 2021, no deaths had occurred among the 14000 staff of the hospital system.

\section{Understanding and improving processes}

Healthcare providers used process mapping and redesign as part of their response to the pandemic. ${ }^{5}$ Flow charts can help to visualise steps in a process. Improvement often involves testing new ideas to simplify or redesign processes to make them more efficient, which can be particularly critical in emergencies when resources are stretched or new processes are needed.

Seventy per cent of respondents to the $Q$ insight survey thought that improvement tools had been used to a great or moderate extent in rapidly reviewing and improving processes and practices. ${ }^{6}$ Figure 3 shows an example from an East London trust which helped visualise, standardise, digitise, and automate the process for ordering personal protective equipment, and illustrates the way in which quality improvement can enable fast responses. In one day, the steps and decisions shown in figure 3 were mapped by staff, who identified a number of steps that required clarification or could be removed. A digital form was created, aligning with the new standard process, tested by one team, and then scaled throughout the organisation.

Improvement tools, such as flow charts, can help clarification of roles and steps at times of major change. With staff increasingly dispersed at home and work, and major changes to clinical work flow, this tool allows team members to create a shared approach to work, clarifying 


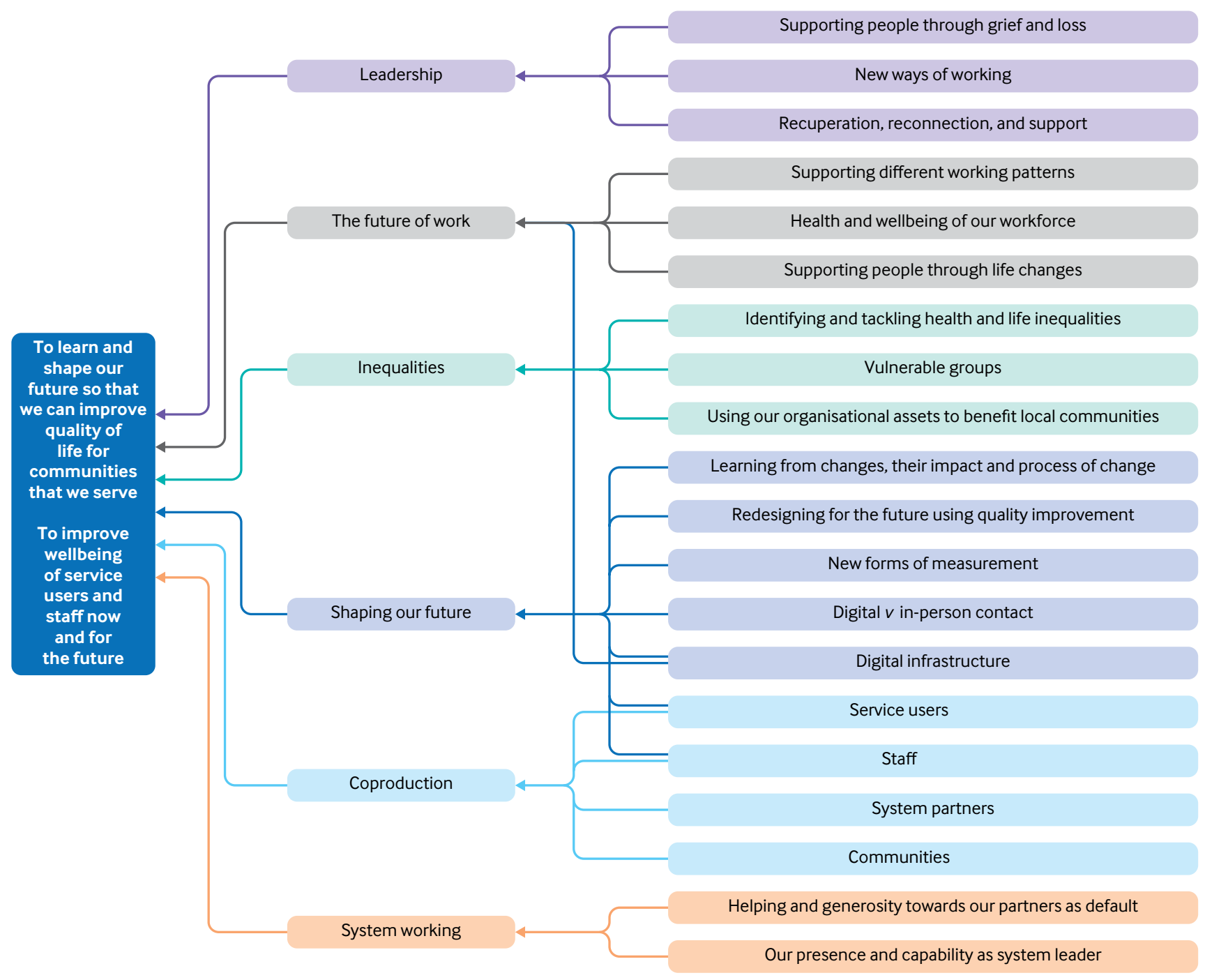

Fig 1 | Example of a driver diagram developed and used at the East London NHS Foundation Trust during the pandemic

uncertainties about roles and simplifying the process.

\section{Measurement to inform decision making}

To ascertain if changes have led to improvement, the data should be studied; this is a key aspect of quality improvement. ${ }^{7}$ Undoubtedly, the pandemic presented challenges, with Q survey respondents describing the difficulties of systematic measurement of improvement during the pandemic, including reduced data collection, high levels of uncertainty about the situation, making it hard to know what to measure, and the speed at which decisions needed to be made. Nevertheless, real time measurement of improvement is particularly helpful when needing to test, learn, and make decisions rapidly.

The Institute for Healthcare Improvement and Associates in Process Improvement have developed modified Shewhart charts to learn from variation in reported deaths in an epidemic. ${ }^{8}$ Albert Einstein Hospital set up a dashboard of key measures that was refreshed daily, to enable the interpretation of variation to guide decision making and to identify the effect of tests of change being carried out. An example of one measure tracked daily is illustrated in figure 4, which helped the hospital to understand the effect of their changes.

\section{Plan-do-study-act (PDSA) cycles to test and scale rapidly}

When a swift response to changing circumstances is required, rapid learning cycles, such as through the PDSA structure, can enable teams to adapt quickly with minimal risk and interruption to clinical work. Having a mechanism to try, and refine, ideas, and ensure that they are plausible before implementing them can support teams in responding to challenges which have no known solution, as described by Fitzsimons. $^{2}$

From the Q insight survey, PDSA cycles were seen to be especially useful owing to the increased need to consider what did and did not work well, especially at the start of the crisis. However, even the rapid iterative nature of PDSAs seemed too arduous for others, with people noting that they were sometimes applied incompletely with some stages missing. One respondent noted that "people are moving too fast to think about the learning generated before making their next plans."

Some organisations found PDSA useful for developing solutions during the pandemic. The Royal National Orthopaedic Hospital used a number of PDSA cycles to support rapid testing, learning, and scaling, to achieve their goal of $80 \%$ of consultations delivered virtually within six weeks. ${ }^{9}$ Surrey and Sussex Healthcare 


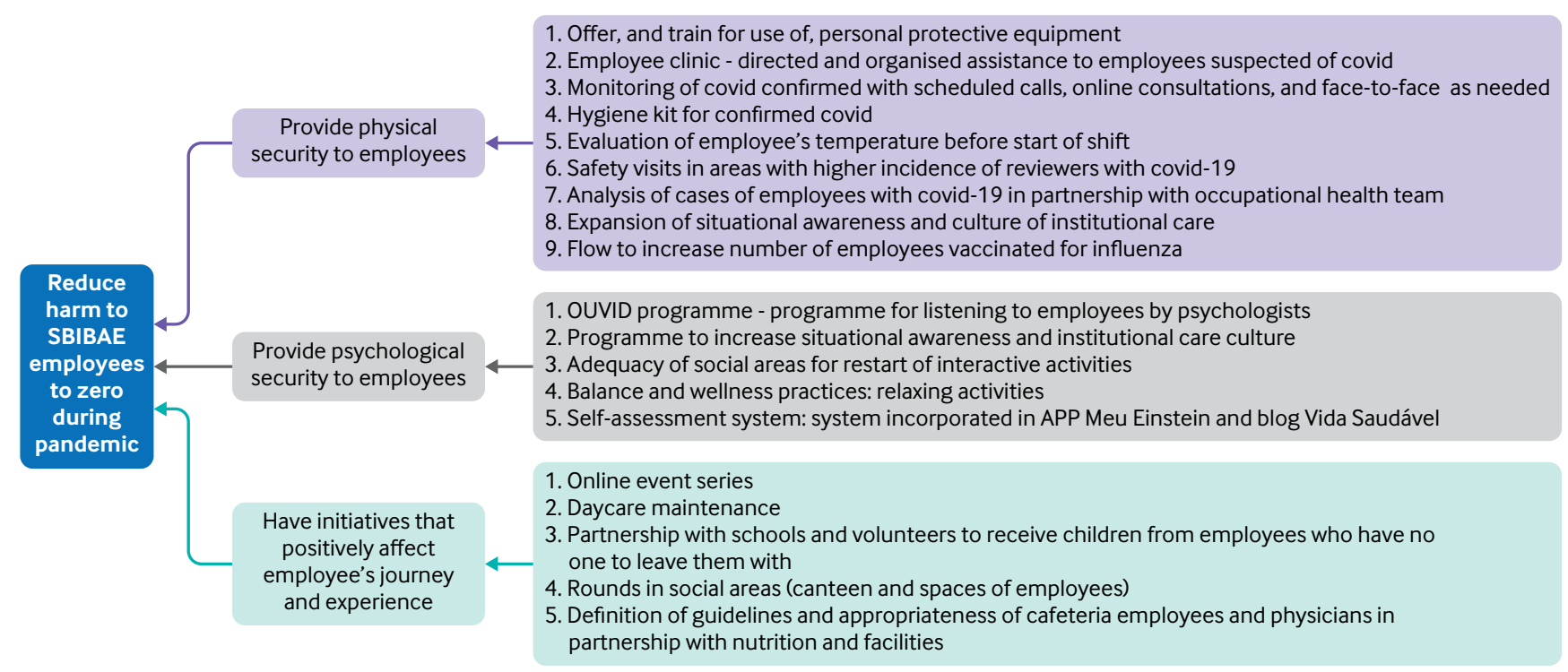

Fig 2 | Driver diagram created by the Albert Einstein Hospital, Brazil, to create a theory of change relevant to the covid-19 pandemic. SBIBAE =Sociedade Beneficente Israelita Brasileira Albert Einstein

Procurement

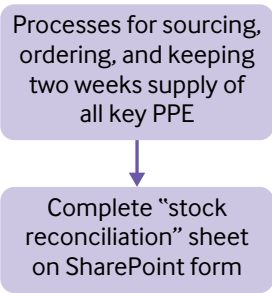

Central fulfilment

Local delivery

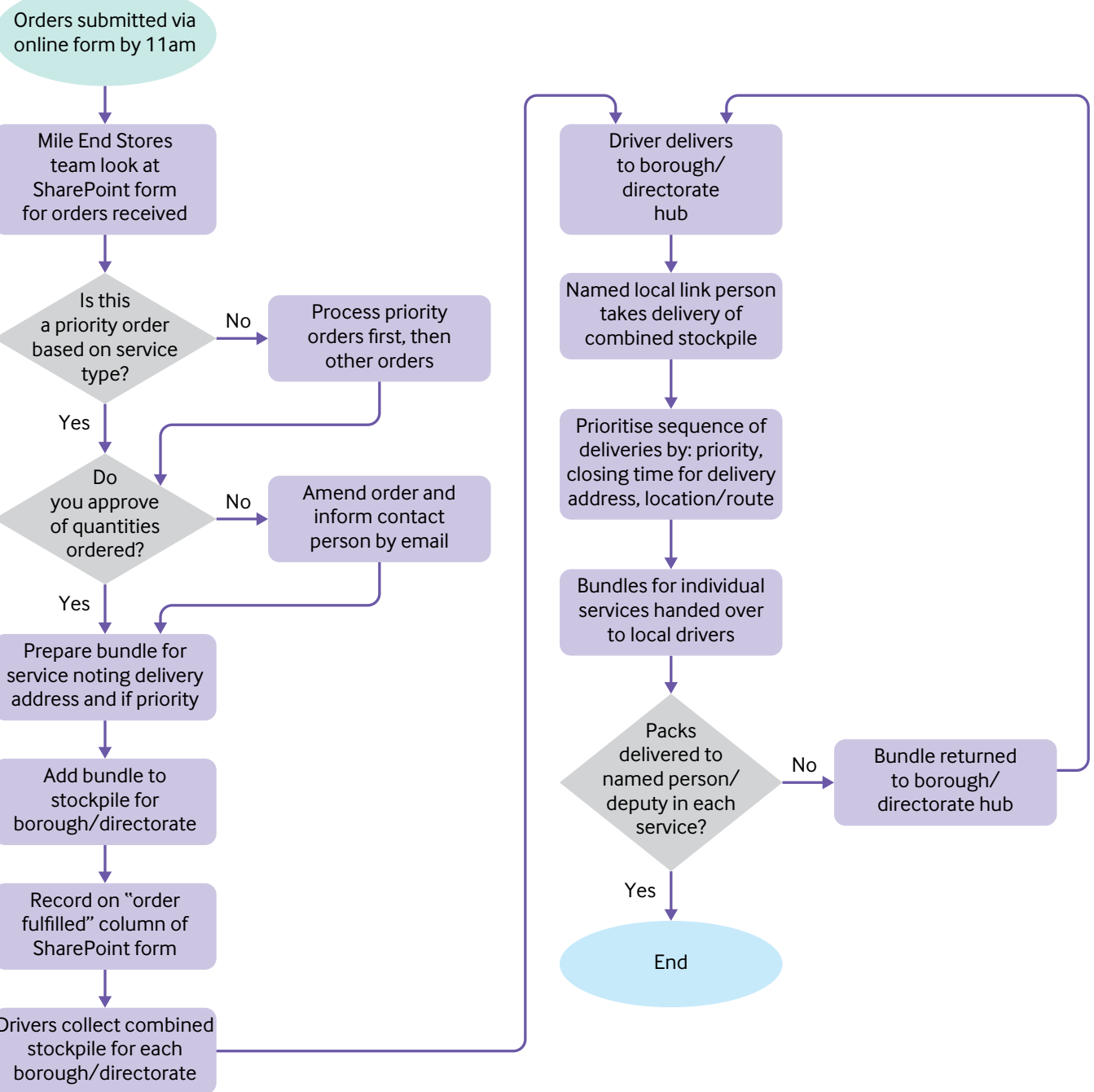




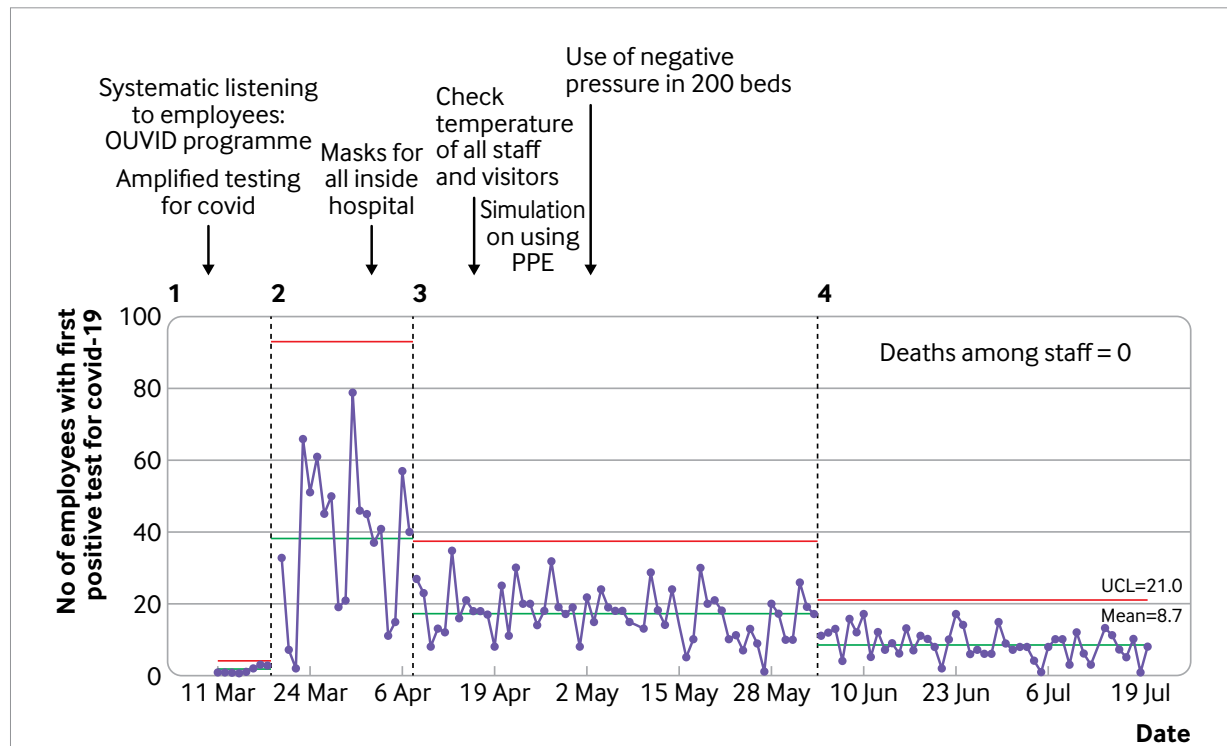

Fig 4 | Data tracked daily during the covid crisis using an annotated statistical process control chart at Albert Einstein Hospital. PPE=personal protective equipment; UCL=upper control limit

NHS Trust used several PDSA cycles to develop and implement a decontamination process which uses ultraviolet light to clean protective hoods worn by staff (consisting of a helmet, face shield, and neck cape). A diverse team developed a range of ideas, eventually discovering that hanging the hood from a disco ball motor and tipping at a $45^{\circ}$ angle allowed the ultraviolet light to reach all areas for decontamination, achieving a $100 \%$ detection rate. ${ }^{10}$

Without a structured approach to experimentation, such as the PDSA cycle, we rely on expertise to design solutions, and implement them without the ability to learn and adapt from small scale to full scale, resulting in a greater risk of failure and less effective solution.

\section{Supporting learning and redesign for the future}

During the acute phase of the pandemic, considerable changes to service provision have taken place and we need to consider which have been helpful and should continue, and which aspects of routine service delivery need to be reinstated. Local and national improvement leaders have used the principles of quality improvement to consider this systematically. ${ }^{11}$

Examples include the musculoskeletal services within the Northern Care Alliance NHS Group, who were supported by the Advancing Quality Alliance through a 60 day innovation cycle to redesign the care pathway (personal communication). At East London Trust, a structured process has been provided for service users to understand the changes made and plan and redesign for the future. ${ }^{12}$

\section{What does this mean for the future of quality improvement in healthcare? \\ Use of methods and measurement}

The methods and tools of quality improvement have been used in a range of different contexts to deal with some of the specific challenges faced during the crisis. Sixty nine per cent of respondents to the $Q$ survey reported that improvement efforts were enabled to a great or moderate extent by reduced bureaucratic constraints, quicker decision making, and fewer financial or procurement hurdles. Some respondents, however, found that the discipline and formal improvement structure was too restrictive during an emergency. Respondents prioritised service delivery over evaluation of the effectiveness of changes. The use of quality improvement often seemed to be characterised by short term goals, and many respondents questioned whether this might undermine the longer term sustainability of changes.

These replies should provoke us to learn how improvement and measurement can be legitimately streamlined while still accelerating testing and learning and reducing demand on clinicians. We have an opportunity to simplify language, avoid differentiating between improvement methods that have the same underpinning scientific roots, and creating too many requirements that stifle activity.

Quality improvement requires proportionate measurement to confidently improve systems and outcomes. We can simplify measurement by using routinely collected data available to teams in a way that allows interrogation and use. Provision of skilled support to teams can help with developing simple measurement plans. Using tools such as safety crosses or manually updated run charts can help teams to connect data collection and interpretation with proposed changes. ${ }^{8}$

\section{Building improvement capability across the system}

Many organisations have seen value in quality improvement tools during the pandemic, but few have demonstrated the full breadth of benefits described by Mondoux et al. ${ }^{4}$ Organisations that have a culture of systematic experimentation and discovery, have trained their workforce, and coached their leaders are more likely to be able to deploy improvement methods effectively and on a larger scale during a crisis. Q members who said their organisation had a well developed approach to improvement before covid were more likely to say that improvement had had an important role during the pandemic. This group was also more likely to use improvement strategically to plan and to collaborate with others. ${ }^{7}$ The unanswered question is whether organisations with this improvement capability achieved better outcomes than others without this expertise.

For many, quality improvement was used in light touch ways, with fuller rigour felt to be too slow during a crisis. This perception of quality improvement is likely to evolve with experience as people become more confident and learn how to apply it to influence their everyday approach to problem solving, with minimal extra work. For example, creating a process map might take an hour but save several hours of wasted activity in a system where roles and steps are not clear. PDSA cycles, conducted well, should save time and reduce waste, by testing rapidly and allowing adaptation to ensure that a solution works well under all known conditions. For those who are newer to the structured approach of quality improvement, their unfamiliarity with the method and tools might not have allowed rapid application to the fast moving environment of the pandemic, when the opportunity for guided learning and reflection is limited.

Quality improvement methods are not just effective for making rapid adaptations in a crisis but, properly supported, can also foster change in the longer term. Organisations with an established improvement culture might be better able 
to leverage the learning from the changes that took place rapidly during the acute phase of the pandemic, to emerge more strategically equipped to improve outcomes in a way that is operationally sustainable.

\section{Leadership for improvement}

The opportunity for leaders is to ensure that the use of improvement tools and approaches during a crisis helps to embed improvement as a strategy for tackling the most complex future challenges. Healthcare staff highlighted increasing autonomy to adapt to the changing circumstances, with $72 \%$ of Q survey respondents approving leadership approaches that have supported local decision making. ${ }^{13}$ Conversely, those respondents who described facing greater challenges in drawing on improvement during the covid-19 pandemic often noted a "command and control" style leadership that stifled testing and innovation. These comments should reinforce our commitment to leadership approaches that provide top-down clarity and encourage bottom-up action.

Developing a deep understanding and application of quality improvement across our health and care systems will require us to continue using improvement tools for daily problem solving, while also using rigorous time limited quality improvement projects for more complex problems. We will need to continue building skills, incentives, and learning mechanisms. Lastly, because modern healthcare is interconnected, many opportunities for improvement will lie in applying quality improvement across boundaries. Thus we need to apply quality improvement over whole systems, as well as within individual organisations.
Competing interests: We have read and understood BMJ policy on declaration of interests and have no relevant interests to declare.

Provenance and peer review: Commissioned; externally peer reviewed.

This article is part of a series commissioned by The $B M /$ based on ideas generated by a joint editorial group with members from the Health Foundation and The BMJ, including a patient/carer. The BM/ retained full editorial control over external peer review, editing, and publication. Open access fees and the quality improvement editor post of The BM/ are funded by the Health Foundation.

Amar Shah, chief quality officer ${ }^{1,3}$

Penny Pereira, Q initiative director

Paula Tuma, quality improvement adviser ${ }^{5}$

${ }^{1}$ East London NHS Foundation Trust, London, UK

${ }^{2}$ Royal College of Psychiatrists, London, UK

${ }^{3}$ University of Leicester, Leicester, UK

${ }^{4}$ Health Foundation, London, UK

${ }^{5}$ Hospital Israelita Albert Einstein, Brazil

Correspondence to: AShah

amarshah@nhs.net

\section{Check for updates}

This is an Open Access article distributed in accordance with the Creative Commons Attribution Non Commercial (CC BY-NC 4.0) license, which permits others to distribute, remix, adapt, build upon this work non-commercially, and license their derivative works on different terms, provided the original work is properly cited and the use is non-commercial. See: http://creativecommons.org/ licenses/by-nc/4.0/.

\section{(9) (1) $($ OPEN ACCESS}

1 Dixon-Woods M, Martin GP. Does quality improvement improve quality?Future Hosp J 2016;3:191-4. doi:10.7861/futurehosp.3-3-191

2 Fitzsimons J. Quality and safety in the time of coronavirus: design better, learn faster. Int J Qual Health Care 2021;33:mzaa051. doi:10.1093/ intqhc/mzaa051.
3 Staines A, Amalberti R, Berwick DM, Braithwaite J, Lachman P, Vincent CA. COVID-19: patient safety and quality improvement skills to deploy during the surge. Int J Qual Health Care 2021;33:mzaa050. doi:10.1093/intqhc/mzaa050

4 Mondoux S, Thull-Freedman J, Dowling S, et al. Quality improvement in the time of coronavirus disease 2019 - a change strategy well suited to pandemic response. CJEM 2020;22:422-5. doi:10.1017/cem.2020.386

5 Hill M, Scott J, Cann H. The role of improvement during the response to covid-19: emergent findings from Q's Insight Survey 2020. Working paper. Health Foundation, 2020. https://s20056.pcdn.co/ wp-content/uploads/2021/05/Q-Insight-surveysummary-Final-draft.pdf

6 Provost L, Bennett B. What's your theory? Driver diagram serves as tool for building and testing theories for improvement. Qual Prog 2015;(Jul): 36-43.

7 Shah A. Using data for improvement. BMI 2019:364:l189. doi:10.1136/bmj.l189

8 Perla R, Provost S, Parry G, Little K, Provost L. Understanding variation in covid-19 reported deaths with a novel Shewhart chart application. Int I Qual Health Care 2021;33:mzaa069. doi:10.1093/ intqhc/mzaa069

9 Gilbert AW, Billany JCT, Adam R, et al. Rapid implementation of virtual clinics due to COVID-19: report and early evaluation of a quality improvement initiative. BMJ Open Qual 2020;9:e000985. doi:10.1136/bmjoq-2020-000985

10 Sansaver J. How a culture of improvement powered health systems' response to covid-19. Health Serv / 2020; Jul 21. https://www.hsj.co.uk/coronavirus/ how-a-culture-of-improvement-powered-healthsystems-response-to-covid-19/7028038.article

11 Rapid learning and improvement during COVID-19: workshop No 1 write-up. https://q.health.org.uk/ news-story/rapid-learning-and-improvement-during covid-19-workshop-1-write-up/

12 East London NHS Foundation Trust. QI essentials: shaping our future. 2020. https://qi.elft.nhs.uk/ qi-essentials-shaping-our-future/

13 Health Foundation. Understanding and sustaining the health care service shifts accelerated by COVID-19. https://www.health.org.uk/publications/ long-reads/understanding-and-sustaining-thehealth-care-service-shifts-accelerated-by-COVID-19

Cite this as: BMJ 2021;373:n928

http://dx.doi.org/10.1136/bmj.n928 